\title{
A Comparison of Cardiac Rehabilitation for Non- Disabling Stroke and Cardiac Conditions: Outcomes and Healthcare Professionals' Perceptions
}

\author{
Authors: \\ *Olive Lennon, ${ }^{1}$ Anne Gallagher, ${ }^{2}$ Hazel Cooney, ${ }^{1}$ Muireann O'Sullivan, \\ Michael Ross, ${ }^{1}$ Jack Smith, ${ }^{1}$ Sean Murphy ${ }^{3}$ \\ 1. School of Public Health, Physiotherapy and Sports Science, University College \\ Dublin, Dublin, UK \\ 2. Cardiac Rehabilitation Services, Mater Misericordiae University Hospital, Dublin, UK \\ 3. Acute Stroke Services, Mater Misericorrdiae University Hospital, Dublin, UK \\ *Correspondence to olive.lennon@ucd.ie
}

Disclosure: $\quad$ The authors have declared no conflicts of interest.

Received: $\quad 17.01 .20$

Accepted: $\quad 19.03 .20$

Keywords: Cardiac rehabilitation, coronary artery disease, stroke.

Citation: $\quad$ EMJ Int Cardiol. 2020;8[1]:26-38.

\section{Abstract}

Purpose: Emerging evidence supports cardiac rehabilitation for transient ischaemic attack (TIA)/nondisabling stroke secondary prevention. This study compares stroke and cardiac participant outcomes following cardiac rehabilitation and examines healthcare professionals' perspectives of cardiac rehabilitation for TIA/non-disabling stroke.

Methods: (1) Retrospective chart review of stroke patients $(n=100)$ referred to cardiac rehabilitation and a matched cardiac group. (2) Semi-structured focus group with healthcare professionals. Independent-t, Mann-Whitney- $U$, and chi-squared tests explored between-group differences. Qualitative data were analysed thematically.

Results: Compared to cardiac counterparts, programme enrolment among stroke patients was lower ( $57 \%$ versus $97 \%$ ) and dropout rates were higher (39\% versus 15\%), $p<0.001$. Healthcare professionals largely attributed this to perceived cognitive impairment. No significant changes were observed between groups in cardiovascular fitness (modified Bruce protocol metabolic equivalent of task [MET] and duration), blood pressure, Hospital Anxiety and Depression Scale, or fasting cholesterol levels, except low-density lipoproteins which reduced more in stroke participants $(p<0.005)$. Both groups made significant improvements post-cardiac rehabilitation in MET (stroke: 2.0 $\pm 1.9 ; p<0.001$ ), (cardiac: 2.2 $\pm 1.6 ; p<0.001$ ); stress test duration (stroke: 2 min $\pm 2.2 ; p<0.001$ ), (cardiac: $2.1 \mathrm{~min} \pm 2.3$; $p<0.001$ ); systolic blood pressure (stroke: $-16.1 \mathrm{mmHg} \pm 20.2 ; \mathrm{p}<0.001$ ), (cardiac: $-11.1 \pm 18.0 ; \mathrm{p}<0.001$ ); low-density lipoprotein (stroke: $-1.2 \mathrm{mmol} / \mathrm{L} \pm 1.0 ; \mathrm{p}<0.001$ ), (cardiac: $-0.7 \pm 0.9 ; \mathrm{p}<0.001$ ); and triglycerides (stroke: $-0.6 \mathrm{mmol} / \mathrm{L} \pm 0.9 ; \mathrm{p}<0.001$ ), (cardiac: $-0.3 \pm 0.9 ; \mathrm{p}=0.002$ ). Significant within-group reductions in total cholesterol were observed in stroke only $(-1.3 \mathrm{mmol} / \mathrm{L} \pm 1.2 ; \mathrm{p}<0.001)$. Reductions in anxiety scores in the Hospital Anxiety and Depression Scale (-0.9; $p=0.01)$ in cardiac participants were not replicated in stroke. Qualitative data identified similar exercise ability in both groups but noted individuals with stroke engaged less in education sessions. 
Conclusion: TIA/non-disabling stroke and cardiac patients made similar physiological and cardiovascular fitness improvements following cardiac rehabilitation. Poor uptake/adherence rates and healthcare professionals' concerns about cognition and engagement warrant investigation.

\section{INTRODUCTION}

Globally, stroke is a leading cause of death and disability. ${ }^{1}$ Recurrence rates are high, with a cumulative 10 -year risk of $39 \%, 2,3$ constituting $25-$ $30 \%$ of all strokes and typically associated with greater severity and disability burden. ${ }^{4,5}$ Current secondary prevention strategies for stroke patients remain largely reliant upon pharmacological therapies ${ }^{6,7}$ for managing cardiovascular disease which are largely attributable to lifestyle risk factors. ${ }^{8,9}$ The INTERSTROKE study identified hypertension and physical inactivity as the top two risk factors for stroke, highlighting important targets for stroke secondary prevention. ${ }^{8}$ The prespecified secondary analysis of the medical arm of the SAMMPRIS trial identified physical activity as an independent predictor for decreasing the likelihood of recurrent strokes, myocardial infarction, or vascular death at 3 years following stroke. ${ }^{10}$ In addition, greater efforts at preventing sedentary behaviour and physical inactivity while promoting exercise training and improving levels of cardiorespiratory fitness have been called for throughout the healthcare system on a worldwide basis to tackle noncommunicable diseases, including cardiovascular disease."

While best practice guidelines in stroke secondary prevention suggest the implementation of multimodal lifestyle interventions for recurrent stroke prevention, the level of evidence to support these recommendations remains limited. ${ }^{6,12}$ Recent research has considered the utility for the cardiac rehabilitation model as a secondary prevention strategy for ischaemic stroke and transient ischaemic attack (TIA), given its proven efficacy in reducing both all cause and cardiac mortality in the coronary artery disease population. ${ }^{13}$ Cardiac rehabilitation has additional proven beneficial effects on lipids, blood pressure, smoking, inflammation, and on psychological risk factors such as depression, anxiety, hostility, and total levels of psychological stress. These benefits have now been extended

to heart failure and peripheral arterial disease. ${ }^{14}$ Evidence from emerging randomised ${ }^{15-17}$ and nonrandomised ${ }^{18-21}$ controlled trials of cardiac rehabilitation and other exercise-based intervention studies broadly modelled on the paradigm ${ }^{22-24}$ indicate that cardiac rehabilitation is feasible and safe for the stroke population and associated with benefits in risk profiles, ${ }^{15-18}$ mortality, ${ }^{25}$ recurrent cardiovascular events, ${ }^{25}$ and hospital readmissions. ${ }^{25}$

However, results from clinical trials do not always translate well to clinical practice. ${ }^{26}$ Challenges to routine implementation, as identified in the literature, include the current adaptability of traditional cardiac rehabilitation programmes to accommodate participants with stroke and its associated sequalae. ${ }^{27}$ Lastly, partnerships between traditional stroke rehabilitation services and cardiac rehabilitation need to be built to operationalise the referral processes required to promote a continuum of care after stroke, inclusive of comprehensive secondary prevention. ${ }^{27}$ This study has two elements aiming to bring new insight to this field by using a mixed methods convergent design to evaluate real world implementation of cardiac rehabilitation after stroke: 1) using retrospective data from a cardiac rehabilitation programme that has piloted referral to cardiac rehabilitation after TIA/ non-disabling stroke at the Mater Misericordiae University Hospital (MMUH), it aims to compare the short-term outcomes following cardiac rehabilitation between individuals with TIA/mild non-disabling stroke and a matched comparator group with cardiac aetiology to establish if comparable changes are made between these groups in routine clinical care; and 2) a focus group from the perspective of healthcare professionals engaged in cardiac rehabilitation assessed the challenges and differences encountered when delivering cardiac rehabilitation to individuals after stroke alongside cardiac counterparts. 


\section{METHODS}

\section{Study Design}

A convergent design with a multistage mixed methods framework was implemented utilising both quantitative and qualitative methodologies. Study 1 consisted of a quantitative, retrospective chart review of data from stroke and cardiac cohorts pre- and post-participation in the cardiac rehabilitation programme. Study 2 was a qualitative semi-structured focus group of healthcare professional stakeholders in cardiac rehabilitation following mild non-disabling stroke. The cardiac rehabilitation programme ran over an 8-week period and participants attended 16 supervised exercise sessions and eight educational and support sessions addressing risk factor reduction and pharmacological compliance as standard.

\section{Ethics}

Ethical approval was granted by the $\mathrm{MMUH}$ Ethics Committee (Institutional Review Board Reference: 1/378/2006) and by the Human Research Ethics Committee, University College Dublin (LS-E-18-176-Lennon Exemption). All participants provided written informed consent.

\section{Procedure}

In Study 1, a retrospective chart review was conducted during October and November 2018 for 100 patients with TIA/non-disabling stroke who had been referred to an 8-week cardiac rehabilitation programme at the $\mathrm{MMUH}$ over the previous 5-year period (2013-2018). Cardiac patients $(n=100)$ matched by age, sex, and the same time period were randomly selected for similar chart review. Baseline data including age, sex, cardiovascular diagnosis, time from cardiovascular event, diagnosis of diabetes, hypertension, family history, alcohol consumption levels, smoking status, physical activity levels, and BMI were extracted. Outcomes extracted pre- and post-cardiac rehabilitation included systolic and diastolic blood pressure, aerobic capacity (MET and duration of a modified Bruce protocol stress test), blood lipids (total cholesterol, low-density lipoprotein [LDL] cholesterol, high-density lipoprotein cholesterol, triglycerides), fasting glucose, and Hospital Anxiety and Depression Scale scores. Programme uptake following referral, dropout rates, and number of exercise and educational classes attended during cardiac rehabilitation were extracted.

Study 2 comprised a focus group, conducted after Study 1 completion. Participants were healthcare professionals affiliated with the cardiac rehabilitation service at the $\mathrm{MMUH}$. Potential participants were volunteers by self-selection, recruited by an information letter sent to relevant staff. Following written consent, each participant provided basic demographic details. The focus group lasted 1 hour and was facilitated by a researcher experienced in qualitative methodologies. The discussion was recorded electronically and an assistant moderator took field notes and recorded nonverbal or emotive responses, or responses generating high agreement. A predesigned question schedule employing introductory, transition, and key questions was employed to guide discussions (adapted from Kreuger). ${ }^{28}$ In conclusion, the moderators provided an oral summary of the discussion content, and participants were provided with the opportunity to respond to and verify these summaries. Focus group transcripts were subsequently sent to participants.

\section{Data Analysis}

The quantitative data from Study 1 were entered on to the Statistical Package for the Social Sciences (SPSS) Version 24.O. Baseline descriptive statistics were summarised. Programme uptake and attendance and dropout rates were compared between stroke and cardiac patients using chi-squared tests. Differences between groups in the number of classes and talks attended were analysed using independent t-tests.

Baseline and change scores pre- and postcardiac rehabilitation were compared between stroke and cardiac groups using independent t-tests for interval or ratio data, Mann-Whitney $U$ tests for nonparametric data, and chi-squared tests for categorical variables. Adjustments for multiple comparisons were made and the significance value was set at $p<0.01$. Subanalysis employed paired t-tests, Wilcoxon signed ranks tests, and chi-squared tests, as appropriate to the data, to identify intragroup changes or explore sex differences. 
Analysis was re-run based on five multiple imputed datasets and missing data postintervention was imputed with Markov Chain Monte Carlo simulations using a fully conditional specification to account for missing items from chart review.

Study 2 recordings were anonymised and transcribed verbatim. Transcripts were reviewed by two independent researchers and thematic analysis conducted utilising transcripts and field notes. Analysis proceeded through stages of familiarisation, thematic framework identification, indexing, charting, mapping, and interpretation, as guided by Pope et al. ${ }^{29}$

Intracoding and intercoding percentage agreement rates were established within and between reviewers. Findings from the qualitative analysis were embedded with the quantitative data using a narrative weaved approach. ${ }^{30}$

\section{RESULTS}

\section{Study 1: Retrospective Chart Review}

A total of $100 \mathrm{TIA} /$ non-disabling stroke patients (modified Rankin score $\leq 2$ ) were referred to cardiac rehabilitation at the time of data collection (October 2018). A corresponding number $(n=100)$ of cardiac referrals were randomly drawn from the same time period. Ten charts were unavailable at the time of the review with a net total of 94 stroke referrals and 96 cardiac referrals reviewed. Baseline descriptive statistics from both groups are presented in Table 1. No statistical differences in baseline characteristics between stroke and TIA populations were identified, except for current alcohol consumption, where the proportion was higher in cardiac participants in comparison to stroke participants $(p=0.04)$. Higher numbers of missing data items in stroke charts in comparison to their cardiac counterparts were identified.

Table 2 summarises programme uptake, dropout, and attendance rates for stroke and cardiac programme participants following referral. A significantly higher proportion of stroke patients did not attend the cardiac rehabilitation service following referral $(p<0.001)$. Similarly, a greater proportion of stroke patients failed to complete the programme $(p=0.002)$, and of those who completed the programme stroke patients attended fewer of the educational support sessions $(p<0.001)$ compared to cardiac patients. No proportional sex differences were identified in programme uptake, dropout rates, or in attendance.

Outcome data pre- and post-cardiac rehabilitation and associated change scores are presented in Table 3. Using the data directly available from chart review, no significant group differences in change scores were noted in outcomes of interest, with the exception of LDL cholesterol which decreased more in the stroke group upon programme completion. The effect size related to this is small (Cohen's $d=0.11$ ). Pooled analysis from the five multiple imputed datasets to account for missing data items identified significant decreases in systolic blood pressure, LDL cholesterol, and triglycerides in the stroke participants when compared to the cardiac group, and again small effect sizes were noted for all these variables (Cohen's $d=0.18-0.40$ ). Cardiac participants in contrast demonstrated significantly greater improvements in diastolic blood pressure, stress test duration, and anxiety reduction, again with small effect sizes noted (Cohen's d=0.17-0.18).

Intragroup analysis identified significant and beneficial changes in systolic blood pressure, aerobic capacity (MET and stress test duration), total cholesterol, and LDL and triglyceride profiles in both patient cohorts. Diastolic blood pressure and the anxiety subscale of the Hospital Anxiety and Depression Scale both improved significantly in cardiac participants, a finding which was not replicated within the stroke group.

\section{Study 2: Focus Group}

Multidisciplinary team members (two cardiac rehabilitation nurses, one clinical psychologist, and one medical consultant) participated in the qualitative focus group discussion. Thematic analysis of their perspectives of cardiac rehabilitation in TIA/non-disabling stroke identified three primary level themes: 1) programme suitability, 2) cognition and mood profiles, and 3) tailoring intervention for stroke. Second level coding identified a further five subcategories under these headings. Intercoding and intracoding percentage agreement were 96\% and $98 \%$, respectively. 
Table 1: Participant descriptive statistics.

\begin{tabular}{|c|c|c|}
\hline Descriptive & Stroke $\mathrm{N}=94$ & Cardiac N=96 \\
\hline & Mean (SD) & Mean (SD) \\
\hline Age (years) & $59.4 \pm 11.2$ & $60.5 \pm 11.9$ \\
\hline \multirow[t]{2}{*}{ Time since event } & $125.4 \pm 50.4$ & $118.6 \pm 98.2$ \\
\hline & $n(\%)$ & $n(\%)$ \\
\hline Male & $70(74.5 \%)$ & 65 (67.7\%) \\
\hline Female & $24(25.5 \%)$ & $31(32.3 \%)$ \\
\hline \multicolumn{3}{|l|}{ Risk factor } \\
\hline \multicolumn{3}{|l|}{ Family history } \\
\hline Stroke & $2(5.6 \%)$ & $4(5.3 \%)$ \\
\hline Cardiac & $17(47.2 \%)$ & $53(70.7 \%)$ \\
\hline Both & $7(19.4 \%)$ & $8(10.7 \%)$ \\
\hline None & $10(27.8 \%)$ & $10(13.3 \%)$ \\
\hline Missing items & 58 & 21 \\
\hline \multicolumn{3}{|l|}{ Hypertension } \\
\hline Yes & $24(49.0 \%)$ & $42(50.0 \%)$ \\
\hline No & $25(51.0 \%)$ & $42(50.0 \%)$ \\
\hline Missing & 45 & 12 \\
\hline \multicolumn{3}{|l|}{ Alcohol consumption* } \\
\hline Current & $25(51.0 \%)$ & $56(66.7 \%)$ \\
\hline Previous & $19(38.8 \%)$ & $16(16.7 \%)$ \\
\hline Never & $5(10.2 \%)$ & $12(12.5 \%)$ \\
\hline Missing items & 45 & 12 \\
\hline \multicolumn{3}{|l|}{ Smoking* } \\
\hline Current & $18(27.3 \%)$ & $15(16.7 \%)$ \\
\hline Previous & $32(48.5 \%)$ & $53(58.9 \%)$ \\
\hline Never & $16(24.2 \%)$ & $22(24.4 \%)$ \\
\hline Missing items & 28 & 6 \\
\hline \multicolumn{3}{|l|}{ Physical activity levels* } \\
\hline Meeting guidelines & $30(69.8 \%)$ & $47(56.6 \%)$ \\
\hline Not meeting guidelines & $13(30.2 \%)$ & $36(43.4 \%)$ \\
\hline$N / D$ & 51 & 13 \\
\hline \multicolumn{3}{|l|}{ Diabetes } \\
\hline Yes & $13(18.3 \%)$ & $16(26.2 \%)$ \\
\hline No & $58(81.7 \%)$ & $45(73.8 \%)$ \\
\hline Missing items & 23 & 35 \\
\hline \multicolumn{3}{|l|}{ BMI } \\
\hline$<18.5$ & $\mathrm{O}(0.0 \%)$ & $0(0.0 \%)$ \\
\hline $18.5-24.9$ & $4(8.0 \%)$ & $18(19.8 \%)$ \\
\hline 25.0-29.9 & $25(50.0 \%)$ & $31(34.1 \%)$ \\
\hline$>30$ & $21(42.0 \%)$ & $42(46.2 \%)$ \\
\hline$N / D$ & 44 & 5 \\
\hline
\end{tabular}

*Self-reported results.

N/D: not documented; SD: standard deviation. 
Table 2: Programme uptake and participation levels.

\begin{tabular}{|c|c|c|c|c|}
\hline \multirow[t]{2}{*}{ Referred to cardiac rehabilitation } & \multirow{2}{*}{$\begin{array}{l}\text { Stroke }(N=94) \\
n(\%)\end{array}$} & \multirow{2}{*}{$\begin{array}{l}\text { Cardiac }(\mathrm{N}=96) \\
\mathrm{n}(\%)\end{array}$} & \multicolumn{2}{|c|}{$\begin{array}{l}\text { Between group } \\
\text { analysis }\end{array}$} \\
\hline & & & $\mathrm{p}$-value & $95 \% \mathrm{Cl}$ \\
\hline Enrolled in the service & $54(57.4 \%)$ & $93(96.9 \%)$ & $<0.001$ & $0.5,0.7$ \\
\hline \multirow[t]{2}{*}{ Completed the programme } & $33(61.1 \%)$ & 79 (84.9\%) & 0.002 & $0.6,0.9$ \\
\hline & Mean (SD) & Mean (SD) & & \\
\hline Number of exercise classes attended & $9.2 \pm 4.9$ & $10.1 \pm 3.9$ & 0.24 & $-2.3,0.6$ \\
\hline $\begin{array}{l}\text { Number of education sessions } \\
\text { attended }\end{array}$ & $4.1 \pm 2.1$ & $5.7 \pm 2.1$ & $<0.001$ & $-2.2,-0.8$ \\
\hline
\end{tabular}

*Self-reported results.

$\mathrm{Cl}$ : confidence interval; SD: standard deviation.

Table 3: Pre- and post-cardiac rehabilitation outcomes for stroke and cardiac groups.

\begin{tabular}{|c|c|c|c|c|c|c|c|c|c|c|c|}
\hline & \multicolumn{4}{|c|}{ Stroke } & \multicolumn{4}{|c|}{ Cardiac } & \multicolumn{3}{|c|}{$\begin{array}{l}\text { Analysis of intergroup } \\
\text { change }\end{array}$} \\
\hline & $\mathrm{n}$ & $\begin{array}{l}\text { Pre-cardiac } \\
\text { rehabilitation }\end{array}$ & $\begin{array}{l}\text { Post-cardiac } \\
\text { rehabilitation }\end{array}$ & Change & $\mathrm{n}$ & $\begin{array}{l}\text { Pre-cardiac } \\
\text { rehabilitation }\end{array}$ & $\begin{array}{l}\text { Post-cardiac } \\
\text { rehabilitation }\end{array}$ & Change & $\mathrm{p}$-value & $\begin{array}{l}95 \% \\
\mathrm{Cl}\end{array}$ & $\begin{array}{l}\text { Effect } \\
\text { size }\end{array}$ \\
\hline \multicolumn{12}{|l|}{ Physiological } \\
\hline $\begin{array}{l}\text { Systolic BP } \\
(\mathrm{mmHg})\end{array}$ & $n=44$ & $140.7 \pm 20.9$ & $124.7 \pm 15.5$ & $-16.1^{*}$ & $n=90$ & $134.9 \pm 19.8$ & $123.8 \pm 16.6$ & $-11.1^{*}$ & 0.651 & $\begin{array}{l}-1.8 \\
11.8\end{array}$ & \\
\hline $\begin{array}{l}\text { Imputed } \\
\text { systolic BP }\end{array}$ & $n=54$ & 141.5 & 125.9 & $-15.6^{*}$ & $n=93$ & 135.0 & 123.9 & $-11.2^{* *}$ & $<0.001^{* *}$ & $\begin{array}{l}-2.1 \\
-6.9\end{array}$ & 0.22 \\
\hline $\begin{array}{l}\text { Diastolic BP } \\
(\mathrm{mmHg})\end{array}$ & $n=45$ & $73.9 \pm 11.9$ & $71.3 \pm 8.9$ & -2.6 & $n=90$ & $73.4 \pm 11.4$ & $68.7 \pm 9.0$ & $-4.7^{*}$ & 0.368 & $\begin{array}{l}-6.6 \\
2.4\end{array}$ & \\
\hline $\begin{array}{l}\text { Imputed } \\
\text { diastolic BP }\end{array}$ & $n=54$ & 74.7 & 72.6 & -2.1 & $n=93$ & 73.1 & 68.6 & $-4.5^{* *}$ & $0.003^{* *}$ & $\begin{array}{l}-4.0 \\
-0.1\end{array}$ & 0.18 \\
\hline \multicolumn{12}{|l|}{$\begin{array}{l}\text { Aerobic } \\
\text { capacity }\end{array}$} \\
\hline METa $^{a}$ & $n=30$ & $6.9 \pm 2.2$ & $8.9 \pm 2.3$ & $2.0^{*}$ & $n=74$ & $7.3 \pm 2.8$ & $9.5 \pm 3.1$ & $2.2^{*}$ & 0.983 & $\begin{array}{l}-0.7 \\
0.7\end{array}$ & \\
\hline Imputed MET & $n=54$ & 7.5 & 9.4 & $1.8^{* *}$ & $n=93$ & 7.4 & 9.6 & $2.2^{* *}$ & 0.031 & $\begin{array}{l}0.02, \\
0.51\end{array}$ & \\
\hline $\begin{array}{l}\text { Stress test } \\
\text { duration } \\
\text { (mins) }\end{array}$ & $n=30$ & $5.5 \pm 2.3$ & $7.5 \pm 2.5$ & $2.0^{*}$ & $n=74$ & $6.1 \pm 2.8$ & $8.2 \pm 2.7$ & $2.1^{*}$ & 0.861 & $\begin{array}{l}-0.9 \\
1.1\end{array}$ & \\
\hline $\begin{array}{l}\text { Imputed } \\
\text { stress test } \\
\text { duration }\end{array}$ & $n=54$ & 6.3 & 8.0 & $1.7^{*}$ & $n=93$ & 6.1 & 8.2 & $2.1^{* *}$ & $0.006^{* *}$ & $\begin{array}{l}0.13 \\
0.76\end{array}$ & 0.17 \\
\hline $\begin{array}{l}\text { Lipids } \\
\text { (mmol/L) }\end{array}$ & & & & & & & & & & & \\
\hline
\end{tabular}


Table 3 continued.

\begin{tabular}{|c|c|c|c|c|c|c|c|c|c|c|c|}
\hline & \multicolumn{4}{|c|}{ Stroke } & \multicolumn{4}{|c|}{ Cardiac } & \multicolumn{3}{|c|}{$\begin{array}{l}\text { Analysis of intergroup } \\
\text { change }\end{array}$} \\
\hline & $\mathrm{n}$ & $\begin{array}{l}\text { Pre-cardiac } \\
\text { rehabilitation }\end{array}$ & $\begin{array}{l}\text { Post-cardiac } \\
\text { rehabilitation }\end{array}$ & Change & $\mathrm{n}$ & $\begin{array}{l}\text { Pre-cardiac } \\
\text { rehabilitation }\end{array}$ & $\begin{array}{l}\text { Post-cardiac } \\
\text { rehabilitation }\end{array}$ & Change & $p$-value & $\begin{array}{l}95 \% \\
\mathrm{Cl}\end{array}$ & $\begin{array}{l}\text { Effect } \\
\text { size }\end{array}$ \\
\hline $\mathrm{TC}$ & $n=41$ & $4.9 \pm 1.0$ & $3.6 \pm 0.6$ & $-1.3^{*}$ & $n=71$ & $5.2 \pm 5.2$ & $3.8 \pm 1.0$ & -1.4 & 0.889 & $\begin{array}{l}-1.7 \\
1.5\end{array}$ & \\
\hline Imputed TC & $n=54$ & 4.6 & 3.7 & -0.9 & $n=93$ & 4.8 & 3.7 & -1.1 & 0.427 & $\begin{array}{l}-0.7 \\
0.3\end{array}$ & \\
\hline LDL & $n=40$ & $3.1 \pm 1.0$ & $1.9 \pm 0.5$ & $-1.2^{*}$ & $n=70$ & $2.7 \pm 0.9$ & $2.0 \pm 0.8$ & $-0.7^{*}$ & $0.005^{*}$ & $\begin{array}{l}-0.2 \\
-0.9\end{array}$ & 0.11 \\
\hline Imputed LDL & $n=54$ & 2.9 & 2.0 & $-0.9^{* *}$ & $n=93$ & 2.6 & 2.0 & $-0.5^{* *}$ & $<0.01^{* *}$ & $\begin{array}{l}-2.3 \\
-0.5\end{array}$ & 0.4 \\
\hline HDL & $n=40$ & $1.0 \pm 0.3$ & $1.1 \pm 0.3$ & 0.6 & $n=71$ & $1.1 \pm 0.4$ & $1.2 \pm 0.4$ & 0.1 & 0.857 & $\begin{array}{l}-0.1 \\
0.1\end{array}$ & \\
\hline Imputed HDL & $n=54$ & 1.1 & 1.2 & $0.1^{*}$ & $n=93$ & 1.1 & 1.2 & 0.1 & 0.01 & $\begin{array}{l}0.1 \\
0.0\end{array}$ & \\
\hline Triglycerides & $n=40$ & $2.0 \pm 1.4$ & $1.4 \pm 1.0$ & $-0.6^{*}$ & $n=70$ & $1.8 \pm 1.3$ & $1.4 \pm 0.9$ & $-0.3^{*}$ & 0.119 & $\begin{array}{l}-0.1 \\
0.6\end{array}$ & \\
\hline $\begin{array}{l}\text { Imputed } \\
\text { triglycerides }\end{array}$ & $n=54$ & 1.9 & 1.4 & $-0.5^{*}$ & $n=93$ & 1.6 & 1.4 & $-0.3^{*}$ & $0.003^{* *}$ & $\begin{array}{l}-0.1 \\
-0.3\end{array}$ & 0.19 \\
\hline $\begin{array}{l}\text { Fasting } \\
\text { glucose } \\
(\mathrm{mmol} / \mathrm{L})\end{array}$ & $n=18$ & $7.5 \pm 8.2$ & $9.3 \pm 11.1$ & 1.7 & $n=34$ & $5.6 \pm 1.6$ & $5.6 \pm 1.2$ & -0.1 & 0.182 & $\begin{array}{l}-4.5 \\
0.9\end{array}$ & \\
\hline $\begin{array}{l}\text { Imputed } \\
\text { fasting } \\
\text { glucose }\end{array}$ & $n=54$ & 7.1 & 8.3 & 1.2 & $n=93$ & 5.7 & 6.2 & 0.5 & 0.066 & $\begin{array}{l}-1.6 \\
0.1\end{array}$ & \\
\hline $\begin{array}{l}\text { HADS } \\
\text { anxiety }\end{array}$ & $n=21$ & $6.0 \pm 4.2$ & $5.9 \pm 4.4$ & 0.2 & $n=65$ & $6.8 \pm 4.4$ & $5.9 \pm 4.0$ & $-0.9 \sim$ & 0.127 & $\begin{array}{l}-0.5 \\
2.8\end{array}$ & \\
\hline $\begin{array}{l}\text { Imputed } \\
\text { HADS anxiety }\end{array}$ & $n=54$ & 6.2 & 6.4 & 0.2 & $n=93$ & 6.8 & 6.2 & -0.6 & $0.002^{*-}$ & $\begin{array}{l}1.3 \\
0.3\end{array}$ & 0.18 \\
\hline $\begin{array}{l}\text { HADS } \\
\text { depression }\end{array}$ & $n=22$ & $4.8 \pm 3.8$ & $3.4 \pm 3.2$ & -0.7 & $n=66$ & $4.4 \pm 3.1$ & $3.7 \pm 3.6$ & -0.7 & 0.93 & $\begin{array}{l}-1.5 \\
1.5\end{array}$ & \\
\hline $\begin{array}{l}\text { Imputed } \\
\text { HADS } \\
\text { depression }\end{array}$ & $n=54$ & 5.1 & 4.2 & -0.8 & $n=93$ & 4.3 & 3.8 & -0.5 & 0.152 & $\begin{array}{l}-0.2 \\
0.8\end{array}$ & \\
\hline
\end{tabular}

BP: blood pressure; Cl: confidence interval; HADS: Hospital Anxiety and Depression Scale; HDL: high-density lipoprotein; LDL: low-density lipoprotein; MET: metabolic equivalent of task; TC: total cholesterol.

a Indicates energy expenditure as a multiple of resting metabolic rate.

* Paired t-test for within group differences $p<0.01$.

$\sim$ Wilcox signed-rank test for intragroup differences $\mathrm{p}<0.01$.

** Independent t-test $\mathrm{p}<0.01$.

*- Mann-Whitney $U$ test $\mathrm{p}<0.01$.

\section{Theme 1: Programme suitability}

As identified in the quantitative data, healthcare professionals noted decreased attendance rates for participants after stroke as well as higher dropout rates and poorer programme compliance. 
Participant 1 (cardiac rehabilitation nurse):

"They didn't always show up [...] they didn't finish. They were the most likely not to turn up for a stress test at the end."

There was an overall consensus that individuals with non-disabling stroke made suitable candidates for cardiac rehabilitation.

Participant 4 (medical consultant):

"Well I believe the cardiac rehab model is equally applicable to stroke patients as it is to coronary heart disease patients, because so many of the risk factors are shared."

The exercise component was highlighted as beneficial for all patients.

\section{Participant 1 (cardiac rehabilitation nurse):}

"I would see that (exercise) as a similarity across cardiac and stroke patients. You can really see how somebody can really get into tunnel vision, so I think the exercise is getting them out of that, breaking that, getting them into social interaction, seeing themselves, monitoring it, improving it [...]."

No difficulty or differences in exercise intensity levels between the two participant groups were observed.

Participant 2 (cardiac rehabilitation nurse): "Are they able to keep up in the same way with their [cardiac] peers? Yes absolutely. No problem."

\section{Theme 2: Cognition and mood profiles}

Cognition was a recurrent theme during the focus group and contrasts between stroke and cardiac patient profiles were stressed.

Participant 1 (cardiac rehabilitation nurse): "I feel very strongly that their cognitive profile is different. They are not as able. I just worry that they're [stroke patients] not keeping up."

Participant 3 (clinical psychologist):

"For them to cognitively get what you're saying, it's different to the cardiac group."

Specific cognitive issues identified included poor recall.
Participant 4 (medical consultant):

"I wonder about capacity to take it in [...] they do not remember what you say to them."

Reduced processing capability was also mentioned.

Participant 3 (clinical psychologist):

"I think with some of the stroke patients [...] there's less awareness, there's less insight, there's more cognitive damage. There's kind of a slower processing."

These issues were thought to affect stroke participants' abilities to participate in the cardiac rehabilitation educational and support components.

Participant 3 (clinical psychologist): "Even in the stress talk they [stroke patients] are not keeping up, and you can't go at that pace necessarily for the wider group. And I have no way of checking in with them without singling them out and saying, 'can you follow what I'm saying'."

Health professionals identified that individuals with stroke considered themselves as being different to their cardiac peers.

Participant 1 (cardiac rehabilitation nurse): "I do wonder whether they feel like there's a difference, they'll often identify themselves as a stroke anytime they'll speak [...] you know there's a bit of 'since l've had the stroke l'll [...]' whereas you know nobody else [cardiac patients] will necessarily mention [their illness] because they'll assume the group is the same."

Differences in mood profiles between stroke and cardiac participants were identified with general agreement that stroke participants tended to be more emotional, frustrated, and have decreased coping abilities, whereas cardiac participants were described as being more anxious in general. Quantitative data support a higher anxiety profile in the cardiac participants on programme commencement but not in depression profiles between the cohorts. 
Participant 1 (cardiac rehabilitation nurse): "[Stroke patients are] not necessarily that kind of high-level anxiety, that cardiac patient type. More almost [...] kind of tearfully, emotionally overwhelmed, and not sure how to cope."

\section{Theme 3: Tailoring the cardiac rehabilitation stroke service}

Health professionals in the focus group agreed that the existing service could be better tailored physically to individuals with non-disabling stroke.

\section{Participant 1 (cardiac rehabilitation nurse):} "You'd have the same exercise piece, but [...] the information [...] you'd be delivering it at a slower pace [for the stroke patients]."

They highlighted that because of the perceived differences in mood and cognitive ability, groupbased sessions were not always optimal.

\section{Participant 3 (clinical psychologist):}

"I think what would work better in terms of the stress talk piece would be an ability to check in [one to one with stroke patients]."

It was discussed that in order to open participation in cardiac rehabilitation to a wider profile of stroke patients, including those with physical disability, additional resources would be required. The limited involvement of therapy professionals in the current programme was considered a barrier to widening participation.

\section{Participant 2 (cardiac rehabilitation}

nurse): "There isn't occupational therapist

involvement and there isn't

\section{physiotherapy involvement."}

More staff, wheelchair accessibility, different/ adaptive equipment, and varying degrees of rehabilitation would be required to cater for more diverse needs of this stroke population. Programme changes and additional supports were identified.

\section{Participant 1 (cardiac rehabilitation nurse):}

'More individualised, supported [...] with appropriate equipment and appropriate layers of people."

\section{DISCUSSION}

This is the first study, to the authors' knowledge, to perform an upfront comparison of outcomes between stroke and cardiac patients after a cardiac rehabilitation programme. Results show that compared to cardiac participants, individuals with TIA/non-disabling stroke acquire similar physiological and physical benefits. Cardiac rehabilitation reduces cardiovascular mortality and morbidity and is cost effective in coronary heart disease treatment. ${ }^{13}$ The parallels in improvement in physiological and aerobic fitness markers between stroke and cardiac patients in this current study show promise for accruing similar benefits for stroke survivors. In a recently published definitive trial in TIA and minor stroke, a support programme using motivational interviewing (without an active exercise component) in comparison to usual care was associated with improved secondary prevention targets at 1 year, but not significant reductions in major vascular events, total vascular events, or all-cause mortality at 3.6 years followup. ${ }^{31}$ Long-term data from similar well conducted multimodal stroke clinical trials including exercise are required.

Aerobic capacity improved in individuals with TIA/non-disabling stroke following 8 weeks of cardiac rehabilitation in this study and the health professionals associated with the programme identified no difficulty with the exercise delivery component. These findings are replicated and extend to those with stroke-related mobility impairments in the literature. A recent systematic review of aerobic programmes for stroke survivors, similar in design to cardiac rehabilitation, identified a small effect size for improved composite aerobic capacity (0.38; confidence interval: 0.27-0.49) following intervention. ${ }^{32}$ Similarly, individuals with mild and moderate stroke-related motor impairments met or exceeded the minimal recommended exercise targets of intensity, duration, and energy expenditure during aerobic and resistance training sessions in cardiac rehabilitation. ${ }^{33}$ Trials of cardiac rehabilitation after stroke in comparison to usual care identified not only significant improvements in aerobic capacity but also in physical activity participation in both stroke and TIA participants. 15,17,22 Noncontrolled studies have identified positive and significant 
changes in aerobic capacity, functional capacity ${ }^{34}$ and in the proportion of individuals categorised in the lowest mortality risk category by stress test result. ${ }^{18}$ In this study both groups made improvements of approximately two MET on fitness testing and this warrants specific comment in secondary prevention strategies. Improvement in cardiorespiratory fitness is noted to be a better prognostic predictor than PA alone ${ }^{11}$ and, albeit in healthy participants, meta-analysis has identified that every one MET increase in cardiorespiratory fitness is associated with a $13 \%$ and $15 \%$ reduction in all-cause and cardiovascular disease/coronary heart disease mortality, respectively. ${ }^{35}$

In the focus group with health professionals, the decreased programme uptake, higher dropout rates, and lower attendance at educational and support sessions by stroke patients were largely attributed to cognitive issues. In acute stroke care a positive correlation was previously identified between attendance rates at an aerobic exercise programme and Functional Independence Measure (FIM) cognitive rating ${ }^{36}$ and severe cognitive deficits identified as a primary cause of ineligibility for cardiac rehabilitation after stroke. ${ }^{37}$ Accommodations for cognitive impairments are not routinely factored into the delivery of the cardiac rehabilitation programmes and cardiac patients are noted to develop only mild cognitive decline 6 years after the event. ${ }^{38}$ However, it is estimated that cognitive impairment occurs in up to $64 \%$ of people who have had a stroke $^{39}$ and even in cases of excellent clinical recovery, high levels of cognitive impairment at 3 months remain. ${ }^{40}$ Furthermore, progressive cognitive decline is described in the years following stroke. ${ }^{41}$ No baseline cognitive screening protocol existed in the cardiac rehabilitation programme reported in this study and confirmation of the cognitive impairment perceived by health professionals is not possible. In future delivery of cardiac rehabilitation after stroke, the inclusion of cognitive screening is recommended to gauge individual capacity and to tailor psychological and education supports appropriately. It is interesting to note in this context, exercise and resistance training for 6 months after stroke has been shown to result in a significant reduction in the proportion of patients meeting the threshold criteria for mild cognitive impairment post-training (65.9\% versus 36.6\%; $p<0.001$ ) and cardiac rehabilitation may have merit as a cognitive intervention after stroke. ${ }^{42}$

In studies where participants were actively recruited, cardiac rehabilitation completion rates reported were higher (80-100\% $)^{17,22}$ in comparison to the $61 \%$ identified in this translational study. Recently published enrolment and completion rates for cardiac rehabilitation after stroke confirm similar completion rates to this study (67\%) when individuals are referred to routine cardiac rehabilitation, but in contrast identified $90 \%$ completion rates when referred to stroke-adapted cardiac rehabilitation programmes. ${ }^{37}$ This warrants future consideration in service development and delivery, given that health professionals in this study considered that individuals with stroke have different profiles to cardiac patients, the stroke survivors self-identify themselves as different to their cardiac counterparts and delivery of educational and support components for stroke was currently deemed suboptimal. Qualitative data from TIA patients who attended conventional cardiac rehabilitation previously identified a downward comparison with individuals who had a cardiovascular event, a need for improved information delivery specific to their risk factors, and addressing the psychological impact of stroke as unmet needs. ${ }^{43}$ Barriers to cardiac rehabilitation attendance by stroke survivors identified in the literature include weather, transport, health issues, and travel distance, ${ }^{44}$ but with many of these issues echoed in cardiac populations. ${ }^{45}$

Mood and emotional disturbances are common in both stroke ${ }^{46}$ and cardiac conditions ${ }^{47}$ and cardiac rehabilitation has established efficacy in reducing the prevalence of anxiety and depression in cardiac participants. ${ }^{48,49}$ Prevalence rates of anxiety and depression in TIA/non-disabling stroke attending cardiac rehabilitation were previously reported in a prospective cohort trial as $39.2 \%$ and $6.5 \%$, respectively and, in line with the findings from this study, the trial identified that completing a cardiac rehabilitation programme was not independently associated with improvement in these domains in stroke..$^{19}$ Conflicting results from previous stroke and cardiac studies identify intragroup reductions in depressive symptoms following cardiac rehabilitation ${ }^{15,49}$ and while reductions in 
depression scores were observed for both groups in the current study, these were not statistically significant.

The health professionals who contributed to the focus group considered cardiac patients to have higher anxiety profiles in comparison to stroke; previously described prevalence rates of anxiety (27\%) and high anxiety (13\%) in cardiac patients attending cardiac rehabilitation match those published for stroke participants., ${ }^{19,48}$ This current study in line with the literature notes significant intragroup reductions in anxiety in cardiac patients following cardiac rehabilitation, but these findings were not replicated in stroke, suggesting there may be subtle differences between the anxiety profiles of both groups or that their responsiveness to group-based management differs. Furthermore, analysis of the imputed dataset identified the reduction in anxiety in cardiac participants to be significantly more than that of participants with stroke. This warrants further investigation.

The high number of missing data items in stroke participants is a noteworthy finding and a limitation in the current study. Several items may have contributed to this finding. The first relates to the differing referral pathways. Cardiac patients are referred either by a structured discharge pathway administered by cardiac rehabilitation staff following surgical intervention or by an online referral mechanism from the acute services hospital information system for nonsurgical patients. Both have a standard proforma and recent test results are available. A small number of cardiac patients from other hospitals and all stroke patients were referred by letter, where the content of the letter was directed by the referring physician. Data routinely collected during the cardiac rehabilitation programme was phased over the initial assessment/orientation appointment and the subsequent cardiac rehabilitation appointment to minimise burden of assessment. The lower attendance rates noted in stroke participants in comparison to cardiac counterparts may have contributed to the higher missing data items noted in this group as a result. Where participants were required to attend phlebotomy services for blood glucose and fasting lipid testing, stroke participants were less likely to follow through with these additional hospital appointments, as noted by health professionals in the focus group conducted who largely attributed this issue to cognitive difficulties. Better integration of individuals with stroke into cardiac rehabilitation has been called for $^{27}$ and a previous out-patient stroke rehabilitation and cardiac rehabilitation partnership proven to be an effective continuum of care. ${ }^{37}$ However, greater understanding of the reasons underpinning poor programme adherence, the support structures required for individuals after stroke in cardiac rehabilitation, and the optimal referral and assessment pathways is still required.

This study as a retrospective chart review, supported by qualitative data representing health professionals' opinions, represents a true depiction of cardiac rehabilitation delivered after TIA/non-disabling stroke in clinical practice. However, a number of limitations must be noted. Both quantitative and qualitative data are drawn from one service and this limits its generalisability. Of note, data collection and analysis were conducted by neutral, independent researchers which limits inherent bias in interpretation of the findings. Similarly, as only individuals with TIA/non-disabling stroke were referred to this service, results cannot be extrapolated to the broader stroke population, encompassing many with stroke related physical disability. A higher proportion of stroke patients than anticipated did not attend the cardiac rehabilitation programme and this, when coupled with missing data items at chart review, limits the interpretation of the findings. While data imputation was conducted to minimise this, further studies with larger numbers are required to support these preliminary findings.

\section{CONCLUSION}

In routine clinical practice, cardiac rehabilitation for individuals with TIA/non-disabling stroke is associated with many of the same fitness and physiological improvements as those in cardiac patients; however, stroke patients are less likely to enrol, complete, or engage with the educational and support components in the programme and do not achieve the same anxiety reduction. Awareness of and screening for cognitive issues after stroke may be required to gauge capacity and tailor interventions appropriately in stroke. Adequately resourced stroke-adapted cardiac rehabilitation may allow wider participation in cardiac rehabilitation and may result in higher enrolment and attendance rates. 


\section{References}

1. Global Burden of Diseases 2016 Stroke Collaborators. Global, regional, and national burden of stroke, 19902016: a systematic analysis for the Global Burden of Disease Study 2016 Lancet Neurol. 2019;18(5):439-58.

2. Mohan KM et al. Risk and cumulative risk of stroke recurrence: a systematic review and meta-analysis. Stroke. 2011;42(5);1489-94.

3. Hankey GJ. Secondary stroke prevention. Lancet Neurol. 2014;13(2):178-94

4. Rothwell PM et al. Change in stroke incidence, mortality, casefatality, severity, and risk factors in Oxfordshire, UK from 1981 to 2004 (Oxford Vascular Study). Lancet. 2004;363(9425):1925-33.

5. Feigin VL et al. Global burden of stroke and risk factors in 188 countries, during 1990-2013: a systematic analysis for the Global Burden of Disease Study 2013. Lancet Neurol. 2016;15(9):913-24.

6. European Stroke Organisation (ESO) executive committee, ESO writing committee. Guidelines for management of ischaemic stroke and Transient Ischaemic Attack 2008. Cerebrovasc Dis. 2008;25(5):457-507.

7. Kernan WN et al. Guidelines for the prevention of stroke in patients with stroke and transient ischemic attack: a guideline for healthcare professionals from the American Heart Association/American Stroke Association. Stroke. 2014;45(7):2160236

8. O'Donnell MJ et al. Global and regional effects of potentially modifiable risk factors associated with acute stroke in 32 countries (INTERSTROKE): a case-control study. Lancet. 2016;388(10046):76175.

9. Turan TN et al. Relationship between risk factor control and vascular events in the SAMMPRIS trial. Neurology. 2017;88(4):379-85.

10. Derdeyn CP et al. Aggressive medical treatment with or without stenting in high-risk patients with intracrania artery stenosis (SAMMPRIS): the final results of a randomised trial. Lancet. 2014:383(9914):333-41.

11. Lavie CJ et al. Sedentary behavior exercise, and cardiovascular health. Circ Res. 2019;124(5):799-815.

12. Quinn TJ et al. Evidence-based stroke rehabilitation: an expanded guidance document from the European Stroke Organisation (ESO) guidelines for management of ischaemic stroke and transient ischaemic attack 2008. J Rehabil Med. 2009;41(2):99-111.

13. Anderson L et al. Exercise-based cardiac rehabilitation for coronary heart disease. Cochrane Database Syst Rev. 2016;1:CD001800

14. Fletcher GF et al.Promoting physical activity and exercise: JACC health promotion series. J Am Coll Cardiol.
2018;72(14):1622-39.

15. Lennon $\mathrm{O}$ et al. A pilot randomized controlled trial to evaluate the benefit of the cardiac rehabilitation paradigm for the non-acute ischaemic stroke population. Clin Rehabil. 2008;22(2);125-33.

16. Lennon $\mathrm{O}$ et al. Clinical trial results - prevention early outcome data from the cardiac rehabilitation adapted for TIA and stroke (CRAFTS) randomized-controlled trial. Poster ESOC-1040 03. European Stroke Organisation Annual Conference, 17-19 April, 2015

17. Kirk $\mathrm{H}$ et al. The cardiac model of rehabilitation for reducing cardiovascular risk factors post transient ischaemic attack and stroke: a randomized controlled trial. Clin Rehabil. 2014;28(4):339-49.

18. Prior $P$ et al. Comprehensive cardiac rehabilitation for secondary prevention after transient ischemic attack or mild stroke: I: feasibility and risk factors. Stroke. 2011;42(11):320713.

19. Prior PL et al. Comprehensive cardiac rehabilitation for secondary prevention after transient ischemic attack or mild stroke: psychological profile and outcomes. J Cardiopulm Rehabil Prev. 2017;37(6):428-36.

20. Cuccurullo SJ et al. Impact of a stroke recovery program integrating modified cardiac rehabilitation on all-cause mortality, cardiovascular performance and functional performance. Am J Phys Med Rehabil. 2019:98(11):953-63.

21. Tang A et al. Feasibility and effects of adapted cardiac rehabilitation after stroke: a prospective trial. BMC Neurol. 2010;10:40.

22. Heron $\mathrm{N}$ et al. Rehabilitation of patients after transient ischaemic attack or minor stroke: pilot feasibility randomised trial of a home-based prevention programme. Br J Gen Pract. 2019;69(687):e706-14.

23. Faulkner $\mathrm{J}$ et al. Effects of early exercise engagement on vascular risk in patients with transient ischemic attack and nondisabling stroke. J Stroke Cerebrovasc Dis. 2016;22(8):e388-96.

24. Faulkner $\mathrm{J}$ et al. The long-term effect of exercise on vascular risk factors and aerobic fitness in those with transient ischaemic attack: a randomized controlled trial. J Hypertens. 2014;32(10):2064-70.

25. Faulkner $\mathrm{J}$ et al. Long-term effect of participation in an early exercise and education program on clinical outcomes and cost implications, in patients with TIA and minor, nondisabling stroke. Transl Stroke Res. 2017;8(3):220-7.

26. Weiss AP et al. Closing the efficacyeffectiveness gap: translating both the what and the how from randomized controlled trials to clinical practice. J Clin Psychiatry. 2009;70(4):446-9.

27. Marzolini S Integrating individuals with stroke into cardiac rehabilitation following traditional stroke rehabilitation: promoting a continuum of care. Can J Cardiol. 2018;34(10 Suppl 2):S240-6

28. Krueger RA. Developing questions for focus groups (1997), London: Sage Publications.

29. Pope C et al. Qualitative research in health care. Analysing qualitative data. BMJ. 2000;320(7227):114-6.

30. Fetters MD et al. Achieving integration in mixed methods designs-principles and practices. Health Serv Res. 2013;48(6pt2):213456.

31. Ahmadi M et al. A support programme for secondary prevention in patients with transient ischaemic attack and minor stroke (INSPiRETMS): an open-label, randomised controlled trial. Lancet Neurol. 2020;19(1):49-60.

32. Regan EW et al. Are aerobic programs similar in design to cardiac rehabilitation beneficial for survivors of stroke? A systematic review and meta-analysis. J Am Heart Assoc. 2019;8(16):e012761.

33. Marzolini S et al. Can individuals participating in cardiac rehabilitation achieve recommended exercise training levels following stroke? $\mathrm{J}$ Cardiopulm Rehabil Prev. 2012;32(3):127-34.

34. Marzolini S et al. Outcomes in people after stroke attending an adapted cardiac rehabilitation exercise program: does time from stroke make a difference? J Stroke Cerebrovasc Dis. 2014;23(6):1648-56

35. Kodama S et al. Cardiorespiratory fitness as a quantitative predictor of all-cause mortality and cardiovascular events in healthy men and women: a meta-analysis. JAMA. 2009;301(19):2024-35

36. Prout EC et al. Patient characteristics that influence enrollment and attendance in aerobic exercise early after stroke. Arch Phys Med Rehabil. 2015;96(5):823-30.

37. Marzolini S et al. Eligibility, enrollment, and completion of exercise-based cardiac rehabilitation following stroke rehabilitation: what are the barriers?. Phys Ther. 2019;100(1):44-56.

38. Selnes OA et al. Cognition 6 years after surgical or medical therapy for coronary artery disease. Ann Neurol. 2008;63(5):581-90.

39. Ya-Ping $\mathrm{J}$ et al. The reciprocal risks of stroke and cognitive impairment in an elderly population. Alzheimers Dement. 2006;2(3):171-8.

40. Jokinen $\mathrm{H}$ et al. Post-stroke cognitive impairment is common even after successful clinical recovery. Eur J Neurol. 2015;22(9):1288-94. 
41. Sachdev PS et al. Progression of cognitive impairment in stroke/ TIA patients over 3 years. J Neurol Neurosurg Psychiatry 2014;85(12):1324-30.

42. Marzolini $\mathrm{S}$ et al. The effects of an aerobic and resistance exercise training program on cognition following stroke. Neurorehabil Neural Repair. 2013;27(5):392-402.

43. Hillsdon $\mathrm{KM}$ et al. A qualitative study exploring patients' experiences of standard care or cardiac rehabilitation post minor stroke and transient ischaemic attack. Clin Rehabil. 2013:27(9):845-53.
44. Marzolini S et al. Factors affecting attendance at an adapted cardiac rehabilitation exercise program for individuals with mobility deficits poststroke. J Stroke Cerebrovasc Dis. 2016;25(1):87-94.

45. Borg $S$ et al. Factors associated with non-attendance at exercise-based cardiac rehabilitation. BMC Sports Sci Med Rehabil. 2019;11(1):13.

46. Kim JS, Choi-Kwon S. Poststroke depression and emotional incontinence: correlation with lesion location. Neurology. 2000:54(9):1805-10.
47. Fan AZ et al. Depression and anxiety associated with cardiovascular disease among persons aged 45 years and older in 38 states of the United States, 2006. Prev Med. 2008;46(5):445-50.

48. Lavie CJ, Milani RV. Prevalence of anxiety in coronary patients with improvement following cardiac rehabilitation and exercise training Am J Cardiol. 2004;93(3):336-9.

49. Milani RV, Lavie CJ. Impact of cardiac rehabilitation on depression and its associated mortality. Am J Med. 20071:120(9):799-806. 